\title{
Experimental Analysis of Color Image Scrambling in the Spatial Domain and Transform Domain
}

\author{
R. Rama Kishore ${ }^{1}$, Sunesh ${ }^{2}$ \\ USICT, Guru Gobind Singh Indraprastha University, New Delhi, India ${ }^{1,2}$ \\ Research scholar and Department of information Technology, MSIT, New Delhi, India ${ }^{2}$
}

\begin{abstract}
This paper proposes two image-scrambling algorithms based on self-generated keys. First color image scrambling method works in the spatial domain, and second, works in the transform domain. The proposed methods cull the $R, G$, and $B$ plane from the color image and scramble each plane separately by utilizing the self-generated keys. The keenness of security of proposed methods is the keys or parameters used in the scrambling process. The exploratory outcomes show that both proposed image scrambling technique performs well in terms of Number of pixel change rate (NPCR), Normalized correlation (NC), Entropy, and time consumed in encoding and decoding. The adequacy of the proposed framework has demonstrated on a data set of five images. In furtherance, the present paper gives a comparative performance analysis between proposed image scrambling methods of spatial domain and transform domain. The proposed paper also tosses some light on the scrambling work reported in the literature.
\end{abstract}

Keywords-Color image scrambling; pixel position modification; spatial domain; Red Green Blue (RGB); transform domain

\section{INTRODUCTION}

With the fast advancement of computer network technology, a large amount of image data had transmitted, quickly and safely over the system that raises different security concerns. For handling the security concern, initially, image encryption had drawn the attention. However, Image encryption is not the same as the content encryption because of some inborn highlights, for example, mass information limit and the great relationship among pixels. In this way, standard cryptographic procedures (DES, IDE, and RSA) are never appropriate for image encryption. Therefore, image scrambling comes out as a vital tool to deal with security concerns. Image scrambling is a technique that realigns all the pixels in an image to various positions to permutated an original image into a new image that is non-recognizable in appearance, confused, and unsystematic.

In literature, The researchers performed scrambling by deploying different concepts like chaos system Fibonacci series, Fibonacci p-code, ASCII code, M-succession, Random number generation, Random shuffling, perfect shuffling, $\mathrm{R}$ prime shuffle, and Sudoku puzzle. Many researchers implemented mainly the concept of chaos. The concept of chaos in image scrambling to achieve diffusion and confusion between image pixels in different ways [1]-[10]. Chaotic system map has many unique properties that classify into two types, namely, 1-D and 2-D. The 1-D chaotic map guide contains a logistic map, Sine map, and tent map. The 2-D chaotic map consists of Arnold, Henon map, and Lorenz system. Background section throws light on the work reported in history.

The Image scrambling methods can be categorized by different ways like based on methodology, based on the domain, based on the type of image. The image scrambling classification is represented by Fig. 1.

Mainly, Image scrambling process uses two types of methodology to accomplish scrambling. One is position shuffling, and other is pixel value modification. The realignment of the pixels is known as pixel position shuffling. Pixel value modification modifies the value of a pixel to achieve image scrambling. Sometimes, the researcher performed pixel value modification alongside pixel position shuffling to improve security. Based on the domain, Image scrambling is classified into two types, termed as spatial domain and transform domain. Image Scrambling in the spatial domain performs scrambling directly on pixels or pixel coordinate. On the other hand, Transform domain scheme performs scrambling by modulating coefficients of the image in the frequency or transform domain [11], [12]. Based on the image type, Image scrambling may be partitioned into two types like grayscale image, color image. In the history, researchers majorly presented various image-scrambling algorithms in the spatial domain for grayscale images in comparison to the transform domain.

In the case of color image scrambling, few researchers reported scrambling algorithm either in the spatial domain or in transform domain. In the last few years, some color image scrambling algorithms in transform domain has been reported. The reported methods carry good efficiency but increased complexity. So, the present paper proposes two color image scrambling methods, one is in spatial domain and second is in transform domain that maintains the efficiency as well as complexity. Both the color image scrambling methods utilizes the concept of self-generated keys. The keys or parameters engaged in scrambling process establish the security of proposed method. Two keys or parameters are adopted by color image scrambling methods. One is self-generated keys that perform scrambling process and second parameter or key controls the complexity of the proposed algorithm. The value of the second parameter may vary; it may be increased or decreased depending upon the requirement. 


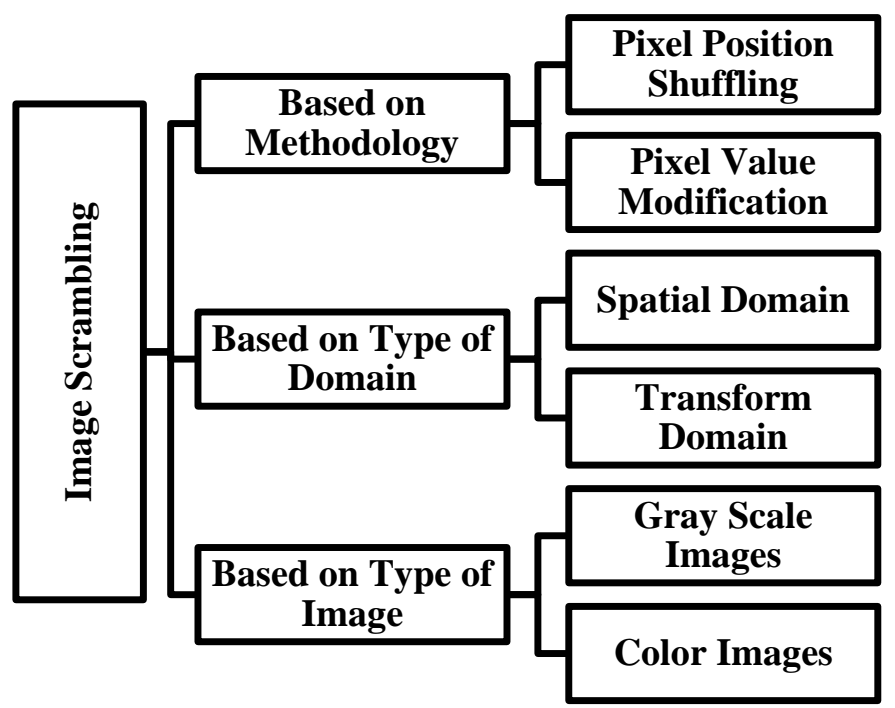

Fig. 1. Classification of Digital Image Scrambling.

Whatever remains of the present paper is sorted out as pursues: Section 2 audits the image scrambling methods reported in the literature. Section 3 presents the proposed color image scrambling in the spatial domain. The proposed color image scrambling algorithm in the transform domain is represented by section 4 . Section 5 illustrates the experimental analysis of both proposed methods. Section 6 discusses the Comparative analysis between both the proposed methods. Finally, Section 7 and Section 8 represent finishing comments and future scope respectively.

\section{RELATED WORK}

The related work section reviews the work related to image scrambling reported in the literature. Majorly the concept of chaos system was exploited to attain diffusion and confusion between image pixels by different ways [1], [2], [13], [3]-[10].

Chaos-based image scrambling can be cultivated by rearranging pixel positions of the image [1], [2], [6], [9]. In this, $\mathrm{Yu}$ et al. proposed an image scrambling system that corporates S-DES with Logistic map [1]. Here, image scrambling exploits the Sensitivity of initial value and randomness trait of the chaotic map that makes a system with larger key quantities. The idea of simplified DES structure makes computing speed quicker. Though, Dong et al. [2] detailed another image scrambling technique which joins the concept of chaos theory with sorting transformation to improve the initial sensitivity of chaotic map. The strong irregularity trait of sorting information makes scrambling effective and secure. Prasad et al. [14] also presented an encryption scheme in which scrambling is achieved by exploiting randomness of chaos map (henon). Dong et al. proposed a color image scrambling that utilizes the inherent features of a chaotic sequence to transform pixel coordinates of RGB image. In 2014, deacons et al. [9] presented a color image encryption method by employing a chaotic map along with the knight's moving rule that transposes pixel between the RGB components. Numerous researchers likewise accomplished chaos-based image scrambling by changing pixel value with position shuffling [5]-[7], [9], [10], [12], [13]. Yangling [3] proposed a scrambling algorithm dependent on chaos sequence and mirror mapping. The author employs the randomness, initial value, and parameter sensitivity traits and generates sequences of real values. Pixel value change is accomplished by playing out an Ex-OR operation between every pixel and real-valued chaotic sequence. Ye[6] exhibited another chaos and bit map based image encryption technique by exploiting pseudo-random property, system parameter, and sensitive dependence on initial conditions and un-periodicity of Chaos map. Position encryption is performed by chaos, and gray value alteration is performed by pixel bit.

Zang et al. [5] reported a bit plane scrambling algorithm with multiple chaotic systems. Arnold and logistic map both are deployed to rearrange the places of pixels in bit-plane. Along with position shuffling, the gray values of the image is also modified. Liu et al. [7] presented space bit plane operation SBPO and chaotic sequence based image encryption scheme, which simultaneously changes the pixel value besides with pixel positions. Based on a chaotic sequence, Author picks every eight pixels from the different segments of the image and form a group. SBPO is performed on each group and build eight pixels with different value. Liu [8] also proposed another image scrambling scheme based on the spatial bit-level map and chaotic sequence that rearranges the image pixels in to1-D logistic chaotic sequences and each pixel in the sequence is decomposed into $\mathrm{k}$ bits for forming a 2-D matrix. The pixel values are modified by applying a spatial bit map on the 2-D matrix. Wang et al. [10] proposed a digital image scrambling algorithm based on chaotic sequence and decomposition and recombination of pixel values. Through decomposition and recombination of pixels, the algorithm scrambles pixel positions and changes pixel values. Liehuang et al. [13]proposed a new cat chaotic mapping based digital image scrambling method for digital image watermarking. The concept of Cat chaotic mapping is utilized to disorder the pixel coordinates of the digital image. Pixel value modification is achieved by performing XOR operation between the certain pixel value of the disordered image and chaotic value.

Sang et al. [15] also reported a block scrambling scheme that by utilizing Arnold and logistic map both. Logistic map generates the sequence, and this sequence is further applied to different Arnold transformed blocks. Xiong et al. [12] proposed a new kind of scrambling transform named local negative base transform (LNBT). The proposed scrambling transform can shuffle the 2-D image with a good enough scrambling performance. Aside from chaos system, the other imperative ideas are used to accomplish encryption of pictures like the Fibonacci series, Fibonacci p-code, ASCII code, Msequence, Random number generation, Random shuffling, perfect shuffling, R prime shuffle and Sudoku puzzle [11], [16]-[25]. In 2004, J. Zou et al. [19] proposed another digital image-scrambling plan with the idea of Fibonacci numbers that makes implementation straightforward with the low computational expense. In 2008, Yicong Zhou et al. [11] presented two image scrambling algorithms by using the idea of Fibonacci p-code that encodes grayscale and a color picture in spatial and transforms domain (DCT). On account of color 
images, YCbCr color space is utilized, and each color segment is extracted at that point, and scrambling is performed on each color segment independently. In this proposed algorithm, $\mathrm{p}$ is taken as a security key to perform scrambling. The proposed technique is a lossless plan that can be utilized for real-life applications. In 2007, Guodong Ye et al. [20] introduced a simple double image scrambling algorithm by exploiting the concept of ASCII code that implements position and pixel value encoding both. In this method, traditional mathematics knowledge is combined with a binary number of computer languages. Decoding of the image is done only when precise keys are known.

Yang Zou et al. [24] presented a Sudoku puzzle based novel image scrambling image algorithm that scrambles the original image both at the pixel and bit level by giving good security. This algorithm uses the property of $\mathrm{N}^{*} \mathrm{~N}$ Sudoku puzzle in which every number from 1 to $\mathrm{N}$ appear only once and maintains the one to one relationship between two Sudoku puzzle. These two Sudoku puzzles are exploited to delineate the original image to a scrambled one. In 2014, H.B. Kekre et al. [26] presented a digital image scrambling method by using qualities of related prime shuffle to give high security. The proposed algorithm is actualized on a block level by partitioning a picture into various blocks. Different R. prime number is used to shuffle row and column of the block. Combination of all r-prime numbers used in block level scrambling is used as a security key to decode the key. Kekre et al. [23] presented an analysis of perfect shuffling characteristics like the downsampling effect, the number of iteration required to get back the original image.

In 2008, [27] proposed a new image scrambling method based on parametric M-sequence. M-sequences are generated by utilizing a series of shift registers that makes decoding of images difficult without knowing the keys. In 2015, M.M. Aziz et al. [21] reported a simple image scrambling algorithm for grayscale images by utilizing traits of random number generation. In this scrambling is achieved by shuffling pixel position without modifying pixel value. In furtherance, Waghulde et al. also throw light on image scrambling methods based on r-prime shuffle, Arnold transformation, and random number generation [28]. In 2008, S.Z.Qin et al. [22] presented a random shuffling based scrambling algorithm that can perform scrambling on the non-equilateral image. The proposed method utilizes the concept of random shuffling to accomplish scrambling by shifting the pixel position of the image.

By inspiring from the above scrambling method, the present paper proposes two secure and efficient color image scrambling methods. One performs in the spatial domain and second performs in the transform domain. Section3 and section4 represent both algorithms, respectively.

\section{Proposed Color Image Scrambling Method in SPATIAL DOMAIN}

In this section, the first color image scrambling method that works in the spatial domain is presented. The proposed image scrambling methods contains two algorithms; one is the scrambling algorithm, and the other is unscrambling algorithm in the spatial domain.

\section{A. Scrambling Algorithm in Spatial Domain}

Step1. Input: Read color image of size M x N.

Step2. Keys: Sf, X(i), Y(j).

Step3. Split the input color image into three planes, namely $R, G$, and $B$.

Step4. Convert each plane into 1-D vector separately. Size of each plane should be equal to $\mathrm{Mx} \mathrm{N}$.

Step5. Keys are generating by using the following rule:

a) $\mathrm{Sf}=(\mathrm{M} \times \mathrm{N}) / 2$;

b) The key X(i) is generated by utilizing the mentioned below lines

For $\mathrm{i}=\mathrm{Sf}: \mathrm{K}$

$\mathrm{X}(\mathrm{i})=\mathrm{X}(\mathrm{Sf}+\mathrm{i})$;

c) The key $Y(j)$ is generated by utilizing the mentioned below lines

For $\mathrm{j}=\mathrm{Sf}: \mathrm{1}$

$\mathrm{Y}(\mathrm{j})=\mathrm{X}(\mathrm{Sf}-\mathrm{j})$;

In this, $\mathrm{i}$ and $\mathrm{j}$ refer to the elements.

Step6. These keys are utilized to obtain $\mathrm{P}(\mathrm{i})$ and $\mathrm{Q}(\mathrm{j})$ sequence, which storing values of $\mathrm{X}$ and $\mathrm{Y}$, respectively.

Step7. Sequence P and Q are applied on each component R, G, and B separately. Sequence P and Q extracts the corresponding Pixel coordinates. The value of Sf is also stored for performing scrambling.

Step8. The process of scrambling is performed by forming a new 1-D vector named as a shuffled vector. The value at $\mathrm{Sf}$ pixel coordinate is kept at the first position of the shuffled vector. Then, the extracted $\mathrm{P}$ sequence is put up at even locations of the shuffled vector, and the value of the extracted $\mathrm{Q}$ sequence is stored at odd positions of the shuffled vector. This entire process is applied to each Component R, G, and B Separately and repeated $\mathrm{Z}$ times. $\mathrm{Z}$ is key or parameter which varies depending upon requirements and controls the efficiency and security of the proposed method.

Step9. Generate scrambled R, G, and B component and concatenate $\mathrm{R}, \mathrm{G}$, and $\mathrm{B}$ component together.

Step10. Produce the scrambled color Image.

\section{B. Unscrambling Algorithm in Spatial Domain}

The proposed color image unscrambling method retrieves the original image from the scrambled image and follows the reverse steps of scrambling. The unscrambling algorithm in the spatial domain is below in the form steps:

Step1. Read the scrambled image

Step2. Split the scrambled image into three planes $R, G$, and $\mathrm{B}$.

Step3. Generate three keys, as described in the scrambling algorithm.

Step4. Generate P and Q sequences corresponding to each plane $\mathrm{R}, \mathrm{G}$, and $\mathrm{B}$. 
Step5. Perform the reverse shuffling for each R, G, and B component. Moreover, repeat the entire shuffling process two times to attain original R, G, and B component.

Step6. Concatenate the extracted R, G, and B plane and generate the original color image.

\section{Proposed Color Image Scrambling Method IN TRANSFORM DOMAIN}

This section presents the second color image-scrambling method, which performs in the transform domain. In this method, host image is pre-processed by transforming an original color image in discrete cosine transform (DCT) frequencies. Here, section A describes the scrambling process of algorithm in transform domain. On other hand, section B presents the unscrambling process of the proposed method.

\section{A. Scrambling Algorithm in Transform Domain}

Step1. Input: Read color image I of size M x N.

Step2. Keys: $\mathrm{Sf}_{\mathrm{D}}, \mathrm{X}_{\mathrm{D}}(\mathrm{i}), \mathrm{Y}_{\mathrm{D}}(\mathrm{j})$.

Step3. Partition the input image I into three planes, namely $\mathrm{R}, \mathrm{G}$, and $\mathrm{B}$.

Step4. Divide each plane R, G, B into the non-overlapping blocks of size $8 \times 8$ and then transform into the discrete transform frequencies.

Step4. Convert the transformed $\mathrm{R}_{\mathrm{T}}, \mathrm{G}_{\mathrm{T}}, \mathrm{B}_{\mathrm{T}}$ planes into 1-D vectors. Size of each transformed plane should be equal to $M$ $\mathrm{x}$ N. rules:

Step5. Keys are constructed by using the below-mentioned

a) $\quad \mathrm{Sf}_{\mathrm{D}}=(\mathrm{M} \times \mathrm{N}) / 2$;

below lines

b) The key $X_{D}(i)$ is generated by utilizing the mentioned

For $\mathrm{i}=\mathrm{Sf}_{\mathrm{D}}: \mathrm{K}$

$\mathrm{X}_{\mathrm{D}}(\mathrm{i})=\mathrm{X}\left(\mathrm{Sf}_{\mathrm{D}}+\mathrm{i}\right)$;

c) The key $Y_{D}(j)$ is generated by utilizing the mentioned below lines

For $\mathrm{j}=\mathrm{Sf}_{\mathrm{D}}: 1$

$Y_{D}(j)=X\left(S f_{D}-j\right)$

In this, $\mathrm{i}$ and $\mathrm{j}$ refer to the elements.

Step6. Procure the sequence $P_{D}(i)$ and $Q_{D}(j)$ by utilizing the values of $\mathrm{Sf}_{\mathrm{D}}, \mathrm{X}_{\mathrm{D}}(\mathrm{i}), \mathrm{Y}_{\mathrm{D}}(\mathrm{j})$ keys.

Step7. Apply Sequence $P_{D}$ and $Q_{D}$ to transformed planes $R_{T}, G_{T}$, and $B_{T}$. Sequence $P_{D}$ and $Q_{D}$ extracts the corresponding Pixel coordinates. The value of $\mathrm{Sf}_{\mathrm{D}}$ is also stored for performing scrambling.

Step8. The process of scrambling is performed by forming a new 1-D vector named as a shuffled vector. The value at $S f_{D}$ pixel coordinate is kept at the first position of the shuffled vector. Then, the extracted $P_{D}$ sequence is put up at even locations of the shuffled vector, and the value of the extracted $\mathrm{Q}_{\mathrm{D}}$ sequence is stored at odd positions of the shuffled vector. This entire process is applied to $R_{T}, G_{T}$, and $B_{T}$ planes and repeated $\mathrm{Zt}$ times. The key $\mathrm{Zt}$ controls the security, complexity of the proposed method.
Step9.Generate scrambled R, G, and B planes and concatenate $\mathrm{R}, \mathrm{G}$, and $\mathrm{B}$ planes together to produce the scrambled image.

$$
\text { Step10. Display the scrambled image. }
$$

\section{B. Unscrambling Algorithm in Transform Domain}

The color image unscrambling algorithm just follows the reverse steps of the scrambling algorithm. The proposed unscrambling algorithm is explained in the mentioned below steps:

\section{Step1. Read the scrambled image}

Step2. Split the scrambled image into three planes R, G, and B.

Step3. Divide each plane R, G, and B into blocks and apply transform on each plane.

Step4. Generate three keys as described in the scrambling algorithm. keys.

Step5 Generate the $\mathrm{P}_{\mathrm{D}}$ and $\mathrm{Q}_{\mathrm{D}}$ sequences by utilizing the

Step6. Perform the reverse shuffling on the transformed $\mathrm{R}_{\mathrm{T}}, \mathrm{G}_{\mathrm{T}}$, and $\mathrm{B}_{\mathrm{T}}$ planes. Moreover, repeat the entire shuffling process $\mathrm{Zt}$ times to attain original $\mathrm{R}, \mathrm{G}$ and $\mathrm{B}$ planes.

Step7. Concatenate the extracted R, G, and B planes and produce the original color image.

Step8. Display the unscrambled image.

\section{EXPERIMENTAL RESULT ANALYSIS}

The performance of presented algorithms is demonstrated through five different measures such as Bit Error Rate (BER), Number of Pixels Change Rate (NPCR), Entropy, Normalized correlation (NC) and Time consumed in scrambling and unscrambling. Both, the proposed scrambling algorithms are implemented on five images, namely; Lena, Peppers, Baboon, Tiger, and House. Experiments were conducted on MATLAB 2015, a platform with core i5 processor, 4GB RAM. The experimental result section consists of two subsections. The first subsection represents the performance analysis of the proposed scrambling method in the spatial domain. The performance analysis of the second proposed scrambling method in transform domain is illustrated by subsection B.

\section{A. Performance Analysis of Proposed Scrambling Method in Spatial Domain}

Initially, the proposed image scrambling method in the spatial domain is evaluated in the form of visual quality, NPCR, BER, Entropy, and NC. The objective of visual scrutinize is to accentuate the visual similarities between the original image and its scrambled image. Table I shows the visual analysis of the proposed image scrambling method in the spatial domain. Table I exhibits original image, scrambled planes (red, green and blue), scrambled and unscrambled looks of Lena, Peppers, Baboon, Tiger and House images respectively. By inferring the Table I, it is concluded that there is no perceptual analogy between scrambled and original image. 


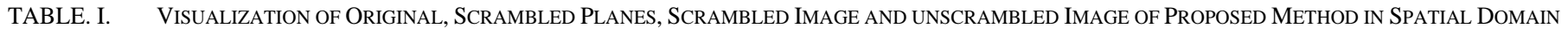
ON LENA, PEPPERS, BABOON, TIGER AND HOUSE IMAGE.

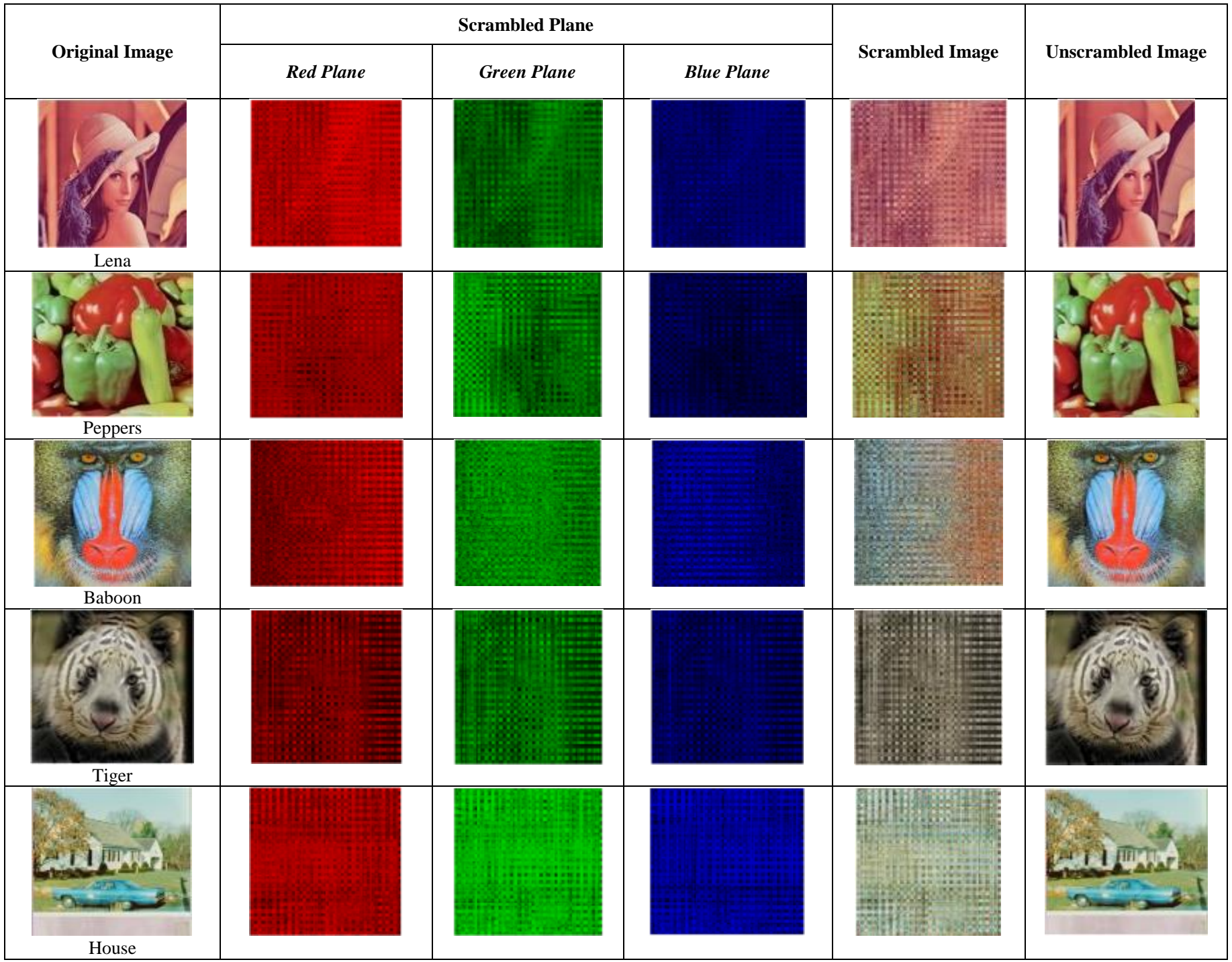

Apart from visual quality analysis, the proposed scrambling method has assessed on some other qualitative parameters that are narrated in Table II and Table III. Table II demonstrates the Number of Pixels Change Rate (NPCR), Bit Error Rate (BER) and Entropy for images Lena, Peppers, Baboon, Tiger, and House.

The parameter NPCR unfolds the strength of image scrambling scheme. Baboon image attains the 99.449 value for NPCR. The proposed watermarking method uses the concept of position shuffling so, the value of UACI indicator comes zero. The proposed method also attains high entropy value in the spatial domain.

For the proposed color image-scrambling scheme, the correlation has been inspected in the two ways. One is on the individual planes (Red. Blue and Green), and other is on the entire image. In both cases, the proposed image scrambling method achieves a high correlation between the original image and the unscrambled image.
Table III delineates the normalized cross-correlation of the red plane, blue plane and green plane of the image individually and combined (all planes) image for Lena, Peppers, Baboon, Tiger, and House images. Time taken in the process of scrambling and unscrambling is also guarded, and Table III depicts the time taken during the scrambling and unscrambling process in seconds.

TABLE. II. NPCR, BER, ENTROPY VALUES OF THE PROPOSED METHOD in SPATial Domain on Lena, PePPERs, Baboon, Tiger and House

\begin{tabular}{|l|l|l|l|}
\hline Image & NPCR & BER & Entropy \\
\hline Lena & 99.2443 & 0 & 7.7749 \\
\hline Peppers & 99.3315 & 0 & 7.756 \\
\hline Baboon & 99.449 & 0 & 7.7624 \\
\hline Tiger & 99.3993 & 0 & 7.7159 \\
\hline House & 99.2704 & 0 & 7.4858 \\
\hline
\end{tabular}


TABLE. III. NC AND TIME CONSUMED IN SCRAMBLING AND UNSCRAMBLING PROCESS OF PROPOSED METHOD IN SPATIAL DOMAIN ON LENA, PEPPERS, BABOON, TIGER AND HOUSE IMAGE

\begin{tabular}{|l|l|l|l|l|l|}
\hline Image & NCR & NCG & NCB & NC & $\begin{array}{l}\text { Time hold for } \\
\text { scrambling and } \\
\text { unscrambling (in Sec) }\end{array}$ \\
\hline Lena & 1 & 1 & 1 & 1 & 2.26 \\
\hline Peppers & 1 & 1 & 1 & 1 & 2.23 \\
\hline Baboon & 1 & 1 & 1 & 1 & 2.35 \\
\hline Tiger & 1 & 1 & 1 & 1 & 2.38 \\
\hline House & 1 & 1 & 1 & 1 & 2.28 \\
\hline
\end{tabular}

The complexity of the proposed can be estimated by looking on the time hold for scrambling and unscrambling. It may be inferred from Table II, Table III, and Table IV that proposed scrambling method in spatial domain performs excellently on all the performance evaluation parameters.

\section{B. Performance Analysis of Proposed Method in Transform Domain}

After analyzing the performance of the proposed method in the spatial domain, the second proposed image-scrambling scheme in the transform domain is examined. In the transform domain, proposed method substantiated on visual quality, Number of Pixels Change Rate (NPCR), Bit Error Rate (BER), Entropy, and Normalized correlation. Table IV illustrates the visual quality of the original image, scrambled red plane, scrambled green plane, and scrambled blue plane, scrambled and unscrambled form Lena, Peppers, Baboon, Tiger, and House images. From Table IV, it may be derived that there is no perceptual similarity between the original and unscrambled image in the transform domain.

TABLE. IV. VisuAlZATION OF ORIGINAL, SCRAMBLED PlanES, SCRAMBLED IMAGE AND UNSCRAMBLED IMAGE OF THE PROPOSED METHOD IN TRANSFORM DOMAIN ON LENA, PEPPERS, BABOON, TIGER AND HOUSE IMAGE.

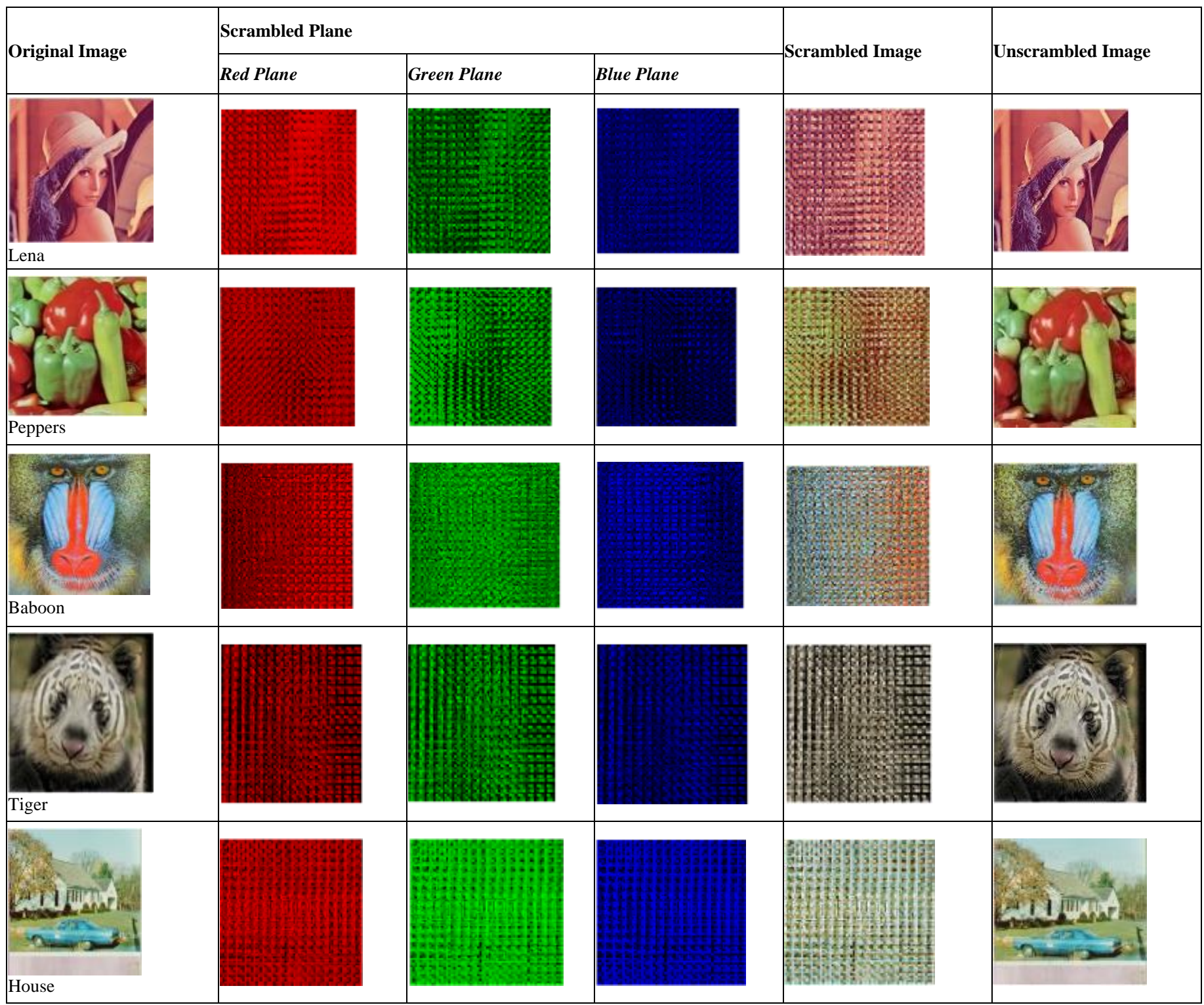


TABLE. V. NPCR, BER, ENTROPY VALUES OF THE PROPOSED METHOD IN THE TRANSFORM DOMAIN ON LENA, PEPPERS, BABOON, Tiger AND HOUSE

\begin{tabular}{|l|l|l|l|}
\hline Image & NPCR & BER & Entropy \\
\hline Lena & 99.1665 & 0.14 & 7.78 \\
\hline Peppers & 99.1041 & 0.23 & 7.79 \\
\hline Baboon & 99.2845 & 0.35 & 7.71 \\
\hline Tiger & 99.3458 & 0.29 & 7.69 \\
\hline House & 99.1922 & 0.27 & 7.64 \\
\hline
\end{tabular}

TABLE. VI. NC AND TIME CONSUMED IN SCRAMBLING AND UNSCRAMBLING PROCESS OF THE PROPOSED METHOD IN TRANSFORM Domain on Lena, PepPers, Baboon, Tiger And House Image

\begin{tabular}{|l|l|l|l|l|l|}
\hline Image & NCR & NCG & NCB & NC & $\begin{array}{l}\text { Time hold in } \\
\text { scrambling and } \\
\text { unscrambling in } \\
\text { Sec }\end{array}$ \\
\hline Lena & 0.9998 & 0.9995 & 1 & 0.9998 & 39.82 \\
\hline Peppers & 1 & 0.9991 & 0.9986 & 0.9992 & 32.69 \\
\hline Baboon & 0.9993 & 0.9998 & 0.9987 & 0.9993 & 48.89 \\
\hline Tiger & 0.9996 & 0.9995 & 0.9992 & 0.9994 & 32.50 \\
\hline House & 0.9998 & 0.9997 & 0.9994 & 0.9996 & 39.76 \\
\hline
\end{tabular}

The other performance evaluation indicators Number of Pixels Change Rate (NPCR), Bit Error Rate (BER), and Entropy are demonstrated in Table V. Table VI manifested that proposed color image scrambling method possesses good NPCR and excellent entropy value on all the test images.

In the transform domain, the proposed scheme is also executed for Normalized correlation and found values very close to 1 . This again proves that the proposed scheme has good correlation value between the original image and unscrambled image. Table VI delineates the normalized crosscorrelation of combined image and the all the three planes of image $(R, G$ and $B)$ individually, and the combined image for Lena, Peppers, Baboon, Tiger, and House. Table VI also depicts the time taken during the scrambling and unscrambling process in seconds.

From Table IV, Table V, and Table VI, it may be concluded that the second proposed color image scrambling method in the transform domain performs well enough on all the evaluation parameters.

\section{COMPARATIVE ANALYSIS AND Discussions}

This section compares the performance of both, proposed color image scrambling methods through four different parameters like visual quality, NPCR, Entropy and NC.

\section{A. Visual Quality Analyses}

Table I and Table IV illustrate the visual quality of the proposed color image scrambling scheme in spatial and transform domain, respectively. It can be deduced from Table I and Table IV that proposed color image scrambling algorithms have no perceptual similarity between the original image and scrambled image, and possess good visual quality in spatial as well as in transform domain on all tested images. From this fact, it may conclude that the proposed color imagescrambling algorithm has good visual quality.

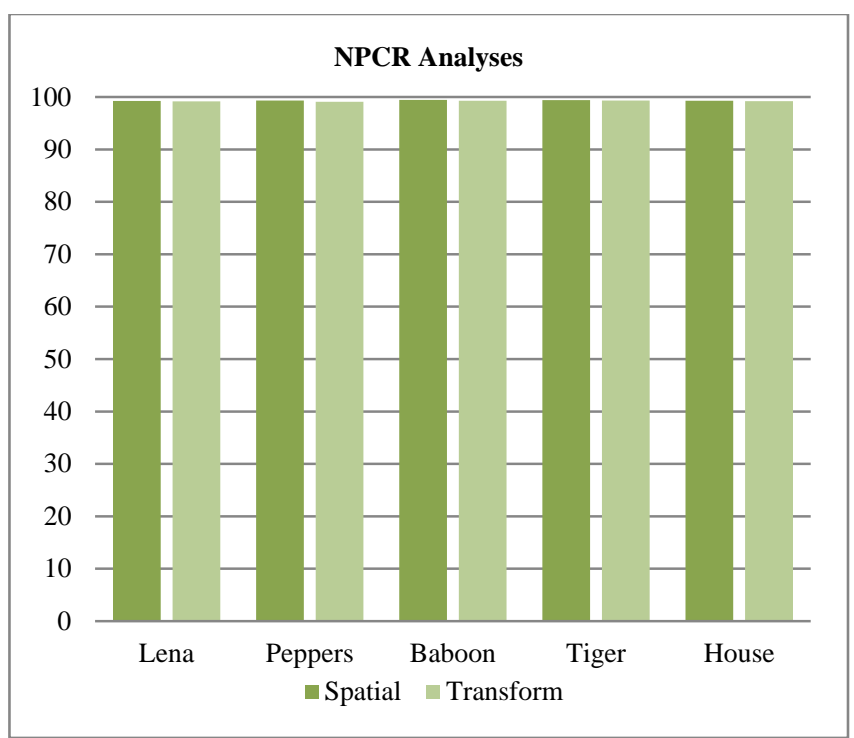

Fig. 2. Comparative Analyses of NPCR in Spatial and Transform domain on Lena, Peppers, Baboon, Tiger, and House Images.

\section{B. NPCR Analyses}

NPCR defines Number of Pixels Change Rate that determines the strength of the image scrambling algorithm. Fig. 2 exemplifies the comparative NPCR analyses of the proposed method for the spatial and the transform domain on Lena, Peppers, Baboon, Tiger, and House images. From Fig. 2, it can identify that the proposed scrambling method has comparable NPCR values for both domains. Comparatively, proposed color image scrambling method attains better value in the spatial domain in comparison to the transform domain. However, it cannot be denied that the proposed scheme has acceptable values for transform domain beyond any doubt. The spatial and transform domain both possess the value of NPCR very close to 100 that is the ideal value. After this comparative analysis, it may conclude that the proposed color image scrambling method has good NPCR.

\section{Entropy Analyses}

Entropy measures the degree of randomness. Table II and Table $\mathrm{V}$ depict the entropy value in spatial and transform domain for the proposed color image scrambling scheme separately. The entropy values are approximate 7.7 for all the tested images in spatial and transform domain that is very close to the highest entropy value can attain by an image. Fig. 3 demonstrates the comparative analyses of entropy measure in spatial and transform domain. From Fig. 3, it can be noticed that the proposed image-scrambling scheme have comparable entropy values for both domains on all tested images. From this comparative entropy analyses, it may be articulated that the proposed color image scrambling method has excellent entropy.

\section{NC Analyses}

In this, $\mathrm{NC}$ defines the correlation between the original image and the unscrambled image. The value of the correlation between the original and the unscrambled image should be high. The value of NC ranges from 0 to 1 . Fig. 4 demonstrates the comparative analyses of $\mathrm{NC}$ between spatial 
and transform domain on Lena, Peppers, Baboon, Tiger, and House Images. The value of NC for spatial domain on all tested images are 1 and transform domain acquires $\mathrm{NC}$ approximated 0.999 . The $\mathrm{NC}$ value attained by the transform domain is close to 1 . Therefore, it can derive that the proposed method has comparable results in terms of $\mathrm{NC}$ for both domains as illustrated in Fig. 3. From Fig. 3, it can conclude that the proposed color image method accomplishes excellent results in terms of NC.

From the above discussions, it can be analyzed that the proposed color image scrambling method has good visual quality, NPCR, Entropy and NC Values in both domains on all the tested images.

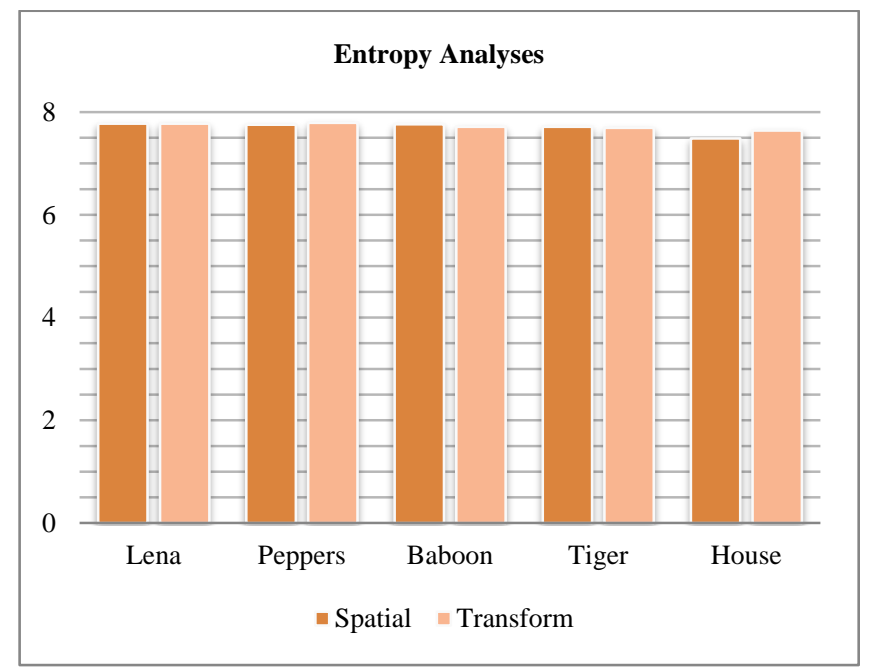

Fig. 3. Comparative Analyses of Entropy in Spatial and Transform Domain on Lena, Peppers, Baboon, Tiger, and House Images.

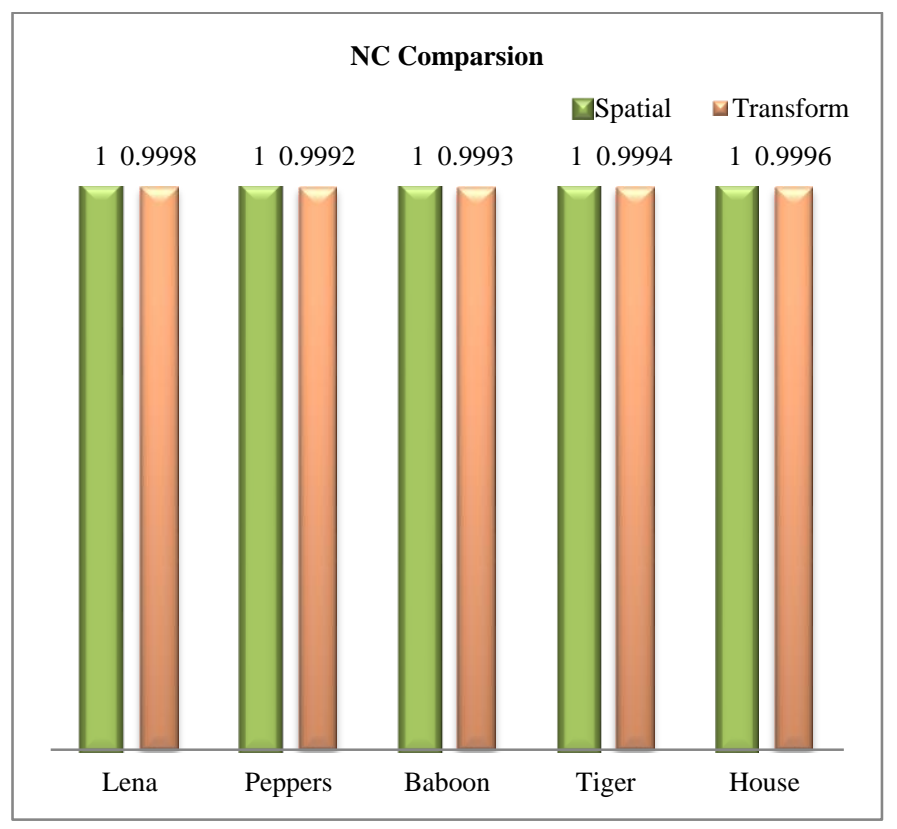

Fig. 4. Comparative Analyses of NC in Spatial and Transform Domain on Lena, Peppers, Baboon, Tiger, and House Images.

\section{CONCLUSION AND FUTURE SCOPE}

The present paper investigates the work associated with digital image scrambling. With this, an effort is put forward in the form of two proposed color image scrambling algorithms that are secure and efficient. The efficiency of the proposed image scrambling method is tested by performing various experiments, as discussed in the experimental results analysis section. The proposed method in the spatial domain is performing excellently in terms of visual quality, NPCR, Entropy, and NC. Whereas in the transform domain, the proposed image scrambling scheme carries excellent results in the form of entropy and performing good in terms of visual quality, NPCR, and NC. In other words, both the proposed color image scrambling algorithms work effectively in their domains.

\section{FUTURE SCOPE}

Performance of proposed color image scrambling method in the transform domain can be enhanced by optimizing the scrambling process.

\section{REFERENCES}

[1] Y. Yu, J. Zhang, H. E. Ren, G. S. Xu, and X. Y. Luo, "Chaotic image scrambling algorithm based on S-DES," in Journal of Physics: Conference Series, 2006, vol. 48, no. 1, p. 349.

[2] L. I. U. Xiangdong, Z. Junxing, Z. Jinhai, and H. Xiqin, "Image scrambling algorithm based on chaos theory and sorting transformation," IJCSNS Int. J. Comput. Sci. Netw. Secur., vol. 8, no. 1, pp. 64-68, 2008.

[3] W. Yanling, "Image scrambling method based on chaotic sequences and mapping," in Education Technology and Computer Science, 2009. ETCS'09. First International Workshop on, 2009, vol. 3, pp. 453-457.

[4] G. Ye, "Image scrambling encryption algorithm of pixel bit based on chaos map," Pattern Recognit. Lett., vol. 31, no. 5, pp. 347-354, 2010.

[5] H. Zhang and R. Cai, "Image encryption algorithm based on bit-plane scrambling and multiple chaotic systems combination," in Intelligent Computing and Integrated Systems (ICISS), 2010 International Conference On, 2010, pp. 113-117.

[6] M. Prasad and K. L. Sudha, "Chaos image encryption using pixel shuffling with henon map," Dimension, vol. 1, p. 50625, 2011.

[7] R. Liu and X. Tian, "A space-bit-plane scrambling algorithm for image based on chaos," J. Multimed., vol. 6, no. 5, pp. 458-466, 2011.

[8] R. Liu, "A New Chaotic Image Scrambling Scheme Based on Spatial Bit-Level Map," in Applied Mechanics and Materials, 2012, vol. 220, pp. 2589-2594.

[9] A.-V. Diaconu, A. Costea, and M.-A. Costea, "Color image scrambling technique based on transposition of pixels between RGB channels using Knight s moving rules and digital chaotic map," Math. Probl. Eng., vol. 2014, 2014.

[10] D. Wang, C.-C. Chang, Y. Liu, G. Song, and Y. Liu, "Digital image scrambling algorithm based on chaotic sequence and decomposition and recombination of pixel values," Int. J. Netw. Secur., vol. 17, no. 3, pp. 322-327, 2015.

[11] Y. Zhou, S. Agaian, V. M. Joyner, and K. Panetta, "Two Fibonacci pcode based image scrambling algorithms," in Image Processing: Algorithms and Systems VI, 2008, vol. 6812, p. 681215.

[12] G. Xiong, S. Zheng, J. Wang, Z. Cai, and D. Qi, "Local Negative Base Transform and Image Scrambling," Math. Probl. Eng., vol. 2018, 2018.

[13] L. L. L. H. Zhu Liehuang Li Wenzhuo, "A Novel Image Scrambling Algorithm for Digital Watermarking Based on Chaotic Sequences," IJCSNS Int. J. Comput. Sci. Netw. Secur., vol. 6, no. 8, pp. 125-130, 2006. 
[14] L. Shou-Dong and X. Hui, "A New Color Digital Image Scrambling Algorithm Based on Chaotic Sequence," in Computer Science \& Service System (CSSS), 2012 International Conference on, 2012, pp. 922-925.

[15] Z. Shang, H. Ren, and J. Zhang, "A block location scrambling algorithm of digital image based on Arnold transformation," in Young Computer Scientists, 2008. ICYCS 2008. The 9th International Conference for, 2008, pp. 2942-2947.

[16] J. Chen, Z. Zhu, C. Fu, L. Zhang, and H. Yu, "Analysis and improvement of a double-image encryption scheme using pixel scrambling technique in gyrator domains," Opt. Lasers Eng., vol. 66, pp. $1-9,2015$.

[17] H. B. Kekre, T. Sarode, P. N. Halarnkar, and D. Mazumder, "Comparative Performance of Image Scrambling in Transform Domain using Sinusoidal Transforms," Int. J. Image Process., vol. 8, no. 2, p. 49, 2014.

[18] J. Chen, Z. Zhu, Z. Liu, C. Fu, L. Zhang, and H. Yu, "A novel doubleimage encryption scheme based on cross-image pixel scrambling in gyrator domains," Opt. Express, vol. 22, no. 6, pp. 7349-7361, 2014.

[19] J. Zou, R. K. Ward, and D. Qi, "A new digital image scrambling method based on Fibonacci numbers," in Circuits and Systems, 2004. ISCAS'04. Proceedings of the 2004 International Symposium on, 2004, vol. 3, pp. III--965.

[20] G. Ye, X. Huang, and C. Zhu, "Image encryption algorithm of double scrambling based on ASCII code of matrix element," in Computational Intelligence and Security, 2007 International Conference on, 2007, pp. 843-847.
[21] M. M. Aziz and D. R. Ahmed, "Simple Image Scrambling Algorithm Based on Random Numbers Generation," Int. J., vol. 5, no. 9, 2015.

[22] S. Liping, Q. Zheng, L. Bo, Q. Jun, and L. Huan, "Image scrambling algorithm based on random shuffling strategy," in Industrial Electronics and Applications, 2008. ICIEA 2008. 3rd IEEE Conference on, 2008, pp. 2278-2283.

[23] H. B. Kekre, T. Sarode, and P. N. Halarnkar, "Study of Perfect Shuffle for Image Scrambling,” Int. J. Sci. Res. Publ., vol. 227.

[24] Y. Zou, X. Tian, S. Xia, and Y. Song, "A novel image scrambling algorithm based on Sudoku puzzle," in Image and Signal Processing (CISP), 2011 4th International Congress on, 2011, vol. 2, pp. 737-740.

[25] P. Praveenkumar, R. Amirtharajan, K. Thenmozhi, and J. B. B. Rayappan, "Triple chaotic image scrambling on RGB--a random image encryption approach," Secur. Commun. Networks, vol. 8, no. 18, pp. 3335-3345, 2015.

[26] H. B. Kekre, T. Sarode, and P. Halarnkar, "Image Scrambling Using RPrime Shuffle on Image and Image Blocks," Int. J. Adv. Res. Comput. Commun. Eng., vol. 3, no. 2, 2014.

[27] Y. Zhou, K. Panetta, and S. Agaian, "An image scrambling algorithm using parameter bases M-sequences," in Machine Learning and Cybernetics, 2008 International Conference on, 2008, vol. 7, pp. 36953698.

[28] D. P. M. M. Dhananjay Santosh Waghulde, "A review on digital image scrambling encryption techniques,” Int. J. Res. Appl. Sci. Eng. Technol., vol. 5, 2017. 